\title{
Strain and strain rate imaging using speckle tracking in acute allograft rejection in children with heart transplantation
}

Sehgal S, Blake JM, Sommerfield J, Aggarwal S. (2015) Strain and strain rate imaging using speckle tracking in acute allograft rejection in children with heart transplantation. Pediatr Transplant, 19: 188-195. DOI: $10.1111 /$ petr.12415.

Abstract: Acute allograft rejection is a major cause of morbidity and mortality following heart transplantation. There is no reliable noninvasive test to diagnose rejection. We aimed to investigate the accuracy of strain by speckle tracking echocardiography in the detection of acute rejection. We identified acute rejection episodes in patients followed at a single transplant center. Data were collected at baseline, during rejection and two follow-up points. Peak systolic radial and circumferential strain at the level of papillary muscles and peak systolic longitudinal strain from apical four-chamber view were analyzed offline. ANOVA was used for comparison between groups. $p$ value $\leq 0.05$ was considered significant. Fifteen rejection episodes were identified. There were no differences in the fractional shortening, LV posterior wall thickness, E/A, septal E/E', septal S', lateral E/E', lateral $\mathrm{S}^{\prime}$, or MPI during rejection, compared to baseline. There was a significant increase in the LV mass during a rejection episode ( $47.5 \mathrm{vs.}$ $\left.34.4 \mathrm{~g} / \mathrm{ht}^{2.7}[\mathrm{p}=0.03]\right)$. The peak systolic radial strain (18.3 vs. 26.5; $\mathrm{p}=0.03)$, longitudinal strain $(-11.7$ vs. $-14.6 ; \mathrm{p}=0.05)$, and circumferential strain $(-14.4$ vs. $-21.7 ; \mathrm{p}=0.05)$ declined significantly during rejection. In conclusion, peak systolic radial, longitudinal and circumferential strain decline and LV mass increases during an episode of rejection.

\author{
Swati Sehgal, Jennifer M. Blake, Julie \\ Sommerfield and Sanjeev Aggarwal \\ Division of Cardiology, Children's Hospital of \\ Michigan, Wayne State University, Detroit, MI, USA
}

Key words: heart transplant - allograft rejection speckle tracking - children

Swati Sehgal, MD, Division of Cardiology,

Department of Pediatrics, Ann and Robert H. Lurie Children's Hospital of Chicago, 225 E Chicago Ave, Chicago, IL 60611, USA

Tel.: +1 3122274425

Fax: +13122279640

E-mail: ssehgal@luriechildrens.org

Accepted for publication 20 November 2014
Heart transplantation is currently an established treatment for end-stage heart failure secondary to cardiomyopathy and repaired as well as irreparable congenital heart disease. Despite advances in the field, a major cause of mortality and morbidity in recipients of heart transplantation is acute allograft rejection (1). Early diagnosis of acute rejection and timely intervention may decrease the risk of progression to hemodynamic compromise and of subsequent allograft vasculopathy. Clinical features of acute rejection are unreliable, with most patients remaining asymp-

\footnotetext{
Abbreviations: ECHO, echocardiographic; ICT, isovolumic contraction time; IRT, isovolumic relaxation time; ISHLT, International Society of Heart and Lung Transplantation; LV, left ventricle; mPCWP, mean pulmonary capillary wedge pressure; MPI, myocardial performance index; mRAP, mean right atrial pressure; ROC, receiver operating characteristic; TDI, tissue Doppler imaging.
}

tomatic with low grade rejection. Endomyocardial biopsy remains the gold standard for diagnosing rejection $(2,3)$, but is invasive, expensive, time-consuming, and may be associated with complications and sampling errors (4). Therefore, the quest for an accurate noninvasive marker of acute rejection continues. Echocardiography is the most commonly used noninvasive imaging modality for screening for suspected rejection. None of the conventional ECHO parameters, though, are sensitive enough to detect asymptomatic or mild episodes of rejection following heart transplantation (5).

The newer ECHO technique of strain imaging by speckle tracking is load and angle independent and free from the influence of respiratory and systolic and diastolic variations (6). It can detect subtle abnormalities of ventricular deformation before an obvious change in systolic functional parameters such as ejection fraction is 
seen (7-9). Studies in the pediatric population have shown abnormalities in strain imaging in the early stages of anthracycline-induced left ventricular dysfunction as well as in obese adolescents without overt ventricular dysfunction (10, 11). It is plausible, therefore, that strain imaging may detect early rejection before overt signs of hemodynamic compromise or other ECHO abnormalities become apparent. Data on the utility of strain and strain rate imaging by speckle tracking in patients with acute rejection following heart transplantation in children or for congenital heart disease are lacking. We undertook this study to investigate the change in strain and strain rate on ECHO during a biopsy-proven rejection episode, in comparison with baseline before and two subsequent time points following treatment of rejection. We hypothesized that left ventricular strain and strain rate would be abnormal during an acute rejection episode in patients with heart transplantation compared to their baseline and would improve following treatment of rejection.

\section{Methods}

This was a retrospective chart review of heart transplantation recipients, who had an episode of acute rejection after one $\mathrm{yr}$ of transplant, defined as ISHLT grade $\geq 2 \mathrm{R}$ on histopathology or evidence of antibody-mediated rejection, defined as the presence of three or more of the following criteria: graft dysfunction, presence of circulating donor-specific antibodies, positive immunofluorescence for $\mathrm{C} 4 \mathrm{~d}$ on the biopsy specimen, and endothelial swelling with interstitial edema on light microscopy (12). Patients were included only if they had an ECHO within $12 \mathrm{~h}$ of the biopsy and were confirmed to be rejection free for at least six months preceding the rejection episode. Patients with poor quality of ECHO images which precluded reliable analysis for strain measurements were excluded. Patients with significant coronary allograft vasculopathy, as determined by coronary angiography on cardiac catheterization, moderate to severe mitral and/or tricuspid valve regurgitation and significant arrhythmias at the time of the ECHO and those who did not have ECHOs at all four data points were also excluded. The study was approved by the Wayne State University Institutional Review Board, and waivers of parental consent and assent were obtained. We initially identified eligible patients from the heart transplant electronic database and then performed a detailed chart review to confirm eligibility criteria.

Demographic and clinical data including age, gender, age at transplant and indications for heart transplantation were abstracted. Details of the rejection episode including clinical presentation, immunosuppressive therapy, rejection grade on biopsy, and treatment received were recorded. The clinical management was at the discretion of the transplant cardiologists. ECHOs were evaluated at four time points for each patient: (i) baseline: preceding the episode of rejection; (ii) rejection episode; (iii) first follow-up data point after treatment of rejection; and (iv) second follow-up after treatment of rejection. At each time point, patients had endomyocardial biopsies within $12 \mathrm{~h}$ of the ECHO, which confirmed acute rejection (time point 2) or absence of rejection (time points 1, 3, and 4).

\section{ECHO data}

A standard ECHO was obtained at our institution during clinic visits and when patients were admitted with suspected acute rejection, using Philips Sono 7500 or IE 33, and stored in the server. We undertook a longitudinal analysis of the following ECHO measures at all four time points in each subject: $\mathrm{M}$ mode measurement for assessment of LV fractional shortening, LV internal dimensions, and LV septal and posterior wall thickness. LV mass was calculated by Devereux formula and powered to height ${ }^{2.7}$. Diastolic indices including peak velocities of early mitral (E) and late phase (A) waves and their ratio (E/A) were measured in apical four-chamber view by pulse-wave Doppler imaging. Similarly, peak early diastolic velocity (E'), late velocity (A'), and systolic velocity (S') were recorded from the septal and lateral mitral valve annulus from apical four-chamber views using TDI. Septal and lateral E/E' ratios were obtained. MPI was calculated using the standard formula: ICT + IRT divided by the ventricular ejection time using pulse Doppler at the junction of aortic and mitral valves in the apical four-chamber view.

Peak systolic longitudinal strain and strain rate from apical 4-chamber views and peak systolic radial and circumferential strain and strain rate from parasternal short axis views at the level of the papillary muscles were calculated offline using vender-independent 2D Cardiac Performance Analysis Software (Tomtec imaging software, Unterschlessheim, Germany). The endocardial borders in apical fourchamber and parasternal short axis views at the level of papillary muscle were manually traced. The papillary muscle and thin trabeculations were ignored while tracing. After the tracing was complete, images were played and manually adjusted if necessary. Peak longitudinal systolic strain and strain rate were obtained in the apical four-chamber view which includes six LV segments and their mean. Similarly, peak systolic radial and circumferential strain were obtained from parasternal short axis view using six segments of the $\mathrm{LV}$ and their mean. Therefore, global strain and strain rate were calculated from six segments in the apical four-chamber (longitudinal strain) and six segments in the parasternal short axis (radial and circumferential strain) view. These digital (DICOM) images were analyzed after selecting the best single beat loop. The speckle tracking strain imaging was analyzed by a single investigator (SS) who was blinded to the results of the biopsy. Other ECHO parameters were analyzed offline by the same investigator using Xcelera software.

\section{Cardiac catheterization data}

mRAP and mPCWP were recorded for all patients at the same four time points (baseline, rejection episode, and two follow-up points). Cardiac catheterization data were also reviewed for evidence of coronary allograft vasculopathy on coronary angiogram, which was an exclusion criterion.

\section{Statistical analysis}

Statistical analysis was performed using SPSS statistical program (version 20; SPSS Inc., Chicago, IL, USA). Data are reported as mean \pm standard deviation for normally 
distributed data and as median (range) for asymmetric data. Repeated-measures ANOVA was used to compare ECHO data at four time points. A p value $\leq 0.05$ was considered significant.

\section{Results}

A total of 82 patients are being followed in our transplant clinic, of which 15 met all inclusion criteria. Demographic and clinical data of the study subjects are summarized in Table 1 . The majority $(\mathrm{n}=10,67 \%)$ of the rejection episodes were cellular, two $(13 \%)$ were humoral, and three $(20 \%)$ were combined rejection episodes. Of the 10 cellular rejection episodes, eight were ISHLT grade $2 \mathrm{R}$ and two were ISHLT grade $3 \mathrm{R}$. Nine of the $15(60 \%)$ patients had symptoms that could be attributed to rejection (resting tachycardia, abdominal pain, emesis, fatigue, shortness of breath), and the remaining six $(40 \%)$ were diagnosed incidentally on endomyocardial biopsy during annual surveillance cardiac catheterization. The primary immunosuppressant regimen was tacrolimus and azathioprine/mycophenolate mofetil for nine patients, tacrolimus and rapamycin for two patients, rapamycin and azathioprine/mycophenolate mofetil for two patients, and cyclosporine and mycophenolate mofetil in two patients. Seven cellular rejection episodes were treated with intravenous methylprednisolone, and three asymptomatic episodes were treated with oral steroids. Thymoglobulin was required in two patients who had evidence of rejection on the first follow-up biopsy after two wk. All humoral and combined rejection episodes were treated with IVIg and plasmapheresis. Three patients received additional treatment with bortezomib $(\mathrm{n}=2)$ or rituximab $(\mathrm{n}=1)$.

\section{ECHO data}

Speckle tracking: Median peak systolic longitudinal strain $(-14.6$ vs. $-11.7, \mathrm{p}=0.05)$ and median peak systolic radial strain (26.5 vs. 18.3, $\mathrm{p}=0.03)$ at the level of papillary muscles were lower during the rejection episode as compared to baseline (Table 2, Figs. 1 and 2). Similarly, median peak systolic circumferential strain declined significantly during rejection $(-21.7$ vs. $-14.4, \quad p=0.05 ; \quad$ Table 2, Fig. 3). There appeared to be improvement in the median peak longitudinal strain, median peak systolic radial strain, and median peak systolic circumferential strain at first and second follow-up, but the change did not reach statistical significance (Table 2). Figs. 1, 2, and 3 depict box and whisker plots of median (IQR) values of peak systolic longitudinal strain, peak systolic radial strain, and peak systolic circumferential strain at the four time points, showing significant changes during the rejection episode, compared to baseline. Conventional ECHO parameters: There was a significant increase in the mean LV mass during the rejection episode $(47.5 \pm 19$ vs. $34.4 \pm 11 \mathrm{~g} /$ $\left.\mathrm{ht}^{2.7}, \mathrm{p}=0.04\right)$, compared to baseline; LV mass during the first and second follow-up points showed no significant change. There was no significant difference in the fractional shortening, LV end diastolic dimensions, or LV posterior wall thickness during the rejection episode, compared to baseline (Table 3). Similarly, diastolic indices such as mitral $\mathrm{E}$ and $\mathrm{A}$ velocities, mitral E/A ratio, septal E/E', lateral E/E', septal S', lateral S', and LV MPI did not differ significantly at any time point, compared to baseline (Table 3 ). Hemodynamic parameters on cardiac catheterization: The mRAP increased significantly during the rejection episode compared to baseline $(4.5 \pm 2.4$ vs. $8.5 \pm 5.1 \mathrm{mmHg}, \mathrm{p}=0.001)$ and decreased following therapy, reaching statistically significant improvement at second followup. Similarly, mPCWP increased during the rejection episode $(8.1 \pm 1.9$ vs. $11 \pm 5, \mathrm{p}=0.04)$ and improved significantly by second follow-up (Table 3).

\section{Discussion}

We evaluated the utility of strain and strain rate using speckle tracking in pediatric heart transplant recipients with acute allograft rejection. Strain analysis by speckle tracking is a newer imaging technique that determines myocardial deformation by a frame-by-frame tracking of speckles. It is reproducible and discriminates between active and passive cardiac movements (6). In addition, longitudinal strain is believed to be relatively independent of maturational changes, an important consideration in the pediatric population (13). Speckle tracking echocardiography for evaluation of myocardial deformation is widely available and adds little to cost.

In our study, there was a significant decline in peak systolic radial and circumferential strain, peak systolic radial and circumferential strain rate, and global longitudinal strain during rejection compared to baseline. Changes in strain imaging using speckle tracking during rejection have been shown in one animal and one adult study previously. In a study in rats with acute rejection, radial strain calculated from the parasternal short axis view at the level of the papillary muscles, peak systolic radial and circumferential strain rate and peak early diastolic radial and cir- 
Fig. 1. Peak systolic longitudinal strain at four time points: baseline, rejection, first and second follow-up. First follow-up: 1.5 months $(0.5$ 5 months) [Median (range)]. Second follow-up: six months (4-12 months) [Median (range)].

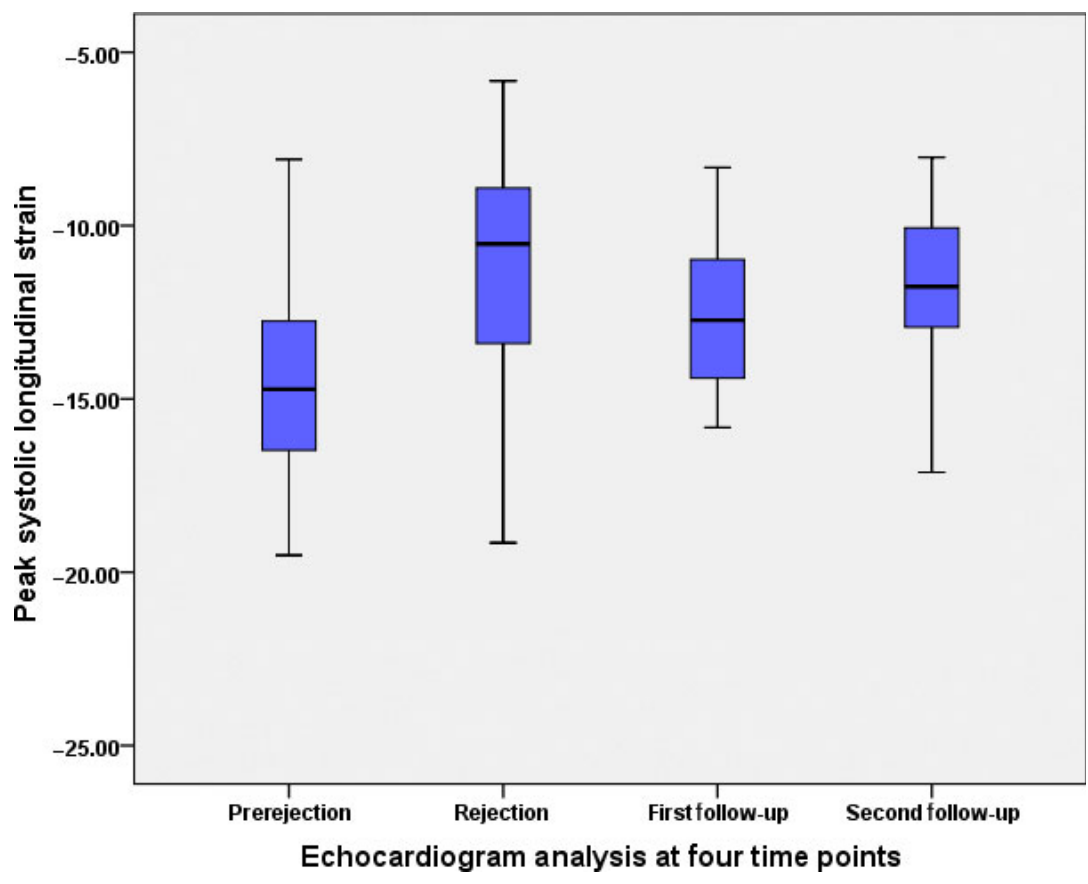

study included only asymptomatic patients, and it is possible that longitudinal strain is the earliest to change, due to the orientation of myocardial fibers.

A few other studies in adults with heart transplantation have shown strain and strain rate abnormalities using tissue Doppler technique during a rejection episode in the absence of any change in the ejection fraction or diastolic ECHO parameters $(16,17)$. Kato et al. analyzed 396 biopsies and echocardiograms in 35 adult patients and found significant differences in the systolic strain, peak systolic strain rate, and peak diastolic strain rate in groups of biopsy-proven rejection $(\geq 1 B)(n=45)$ and rejection-negative patients (16). On multivariate analysis, systolic strain was a strong predictor of rejection, with a cutoff value of $-27.4 \%$ having a predictive accuracy of $82.3 \%$. Marciniak et al. evaluated 31 consecutive adult heart transplant recipients who underwent 106 endomyocardial biopsies and confirmed that longitudinal and radial peak systolic strain and strain rate were significantly decreased in those with grade $\geq 1 \mathrm{~B}$ rejection. Similarly, Roshanali et al. analyzed peak systolic strain and time to systole at three myocardial segments - RV base, interventricular septum, and lateral LV base on 50 ECHOs in 38 patients. Lateral LV base peak strain and time to systole were decreased in patients with rejection, compared to those without rejection (17). However, all these studies used tissue Doppler technique for calculation of strain and strain rate, which is angle dependent and unidimensional (18). 


\section{Sehgal et al.}

Table 2. Comparison of the strain and strain rate between baseline, rejection, and two follow-up points

\begin{tabular}{|c|c|c|c|c|c|}
\hline Parameter & $\begin{array}{l}\text { Baseline } \\
\text { Mean (SD) }\end{array}$ & $\begin{array}{l}\text { Rejection } \\
\text { Mean (SD) }\end{array}$ & $\begin{array}{l}\text { F/u } 1 \\
\text { Mean (SD) }\end{array}$ & $\begin{array}{l}\text { F/u } 2 \\
\text { Mean (SD) }\end{array}$ & p \\
\hline Peak systolic radial strain & $26.5(10)$ & $18.3(9.5)$ & $20.5(8.1)$ & $23.9(10.4)$ & 0.03 \\
\hline Peak systolic longitudinal strain & $-14.6(3)$ & $-11.7(4.3)$ & $-12.7(3.7)$ & $-12.2(2.9)$ & 0.05 \\
\hline Peak systolic circumferential strain & $-21.7(5.4)$ & $-14.4(6.3)$ & $-17.5(4.2)$ & $-17.7(5.7)$ & 0.001 \\
\hline Peak systolic radial strain rate (/s) & $1.8(0.6)$ & $1.4(0.5)$ & $1.7(0.7)$ & $1.6(0.6)$ & 0.05 \\
\hline Peak systolic longitudinal strain rate (/s) & $-0.9(0.6)$ & $-0.9(0.3)$ & $-1.1(0.7)$ & $-1(0.2)$ & 0.8 \\
\hline Peak systolic circumferential strain rate (/s) & $-1.7(0.5)$ & $-1.2(0.5)$ & $-1.4(0.3)$ & $-1.5(0.5)$ & 0.007 \\
\hline
\end{tabular}

F/u 1: First follow-up: 1.5 months (0.5-5 months) from rejection episode [Median (range)].

F/u 2: Second follow-up: six months (4-12 months) from rejection episode [Median (range)].

Numbers in bold are statistically significant results.
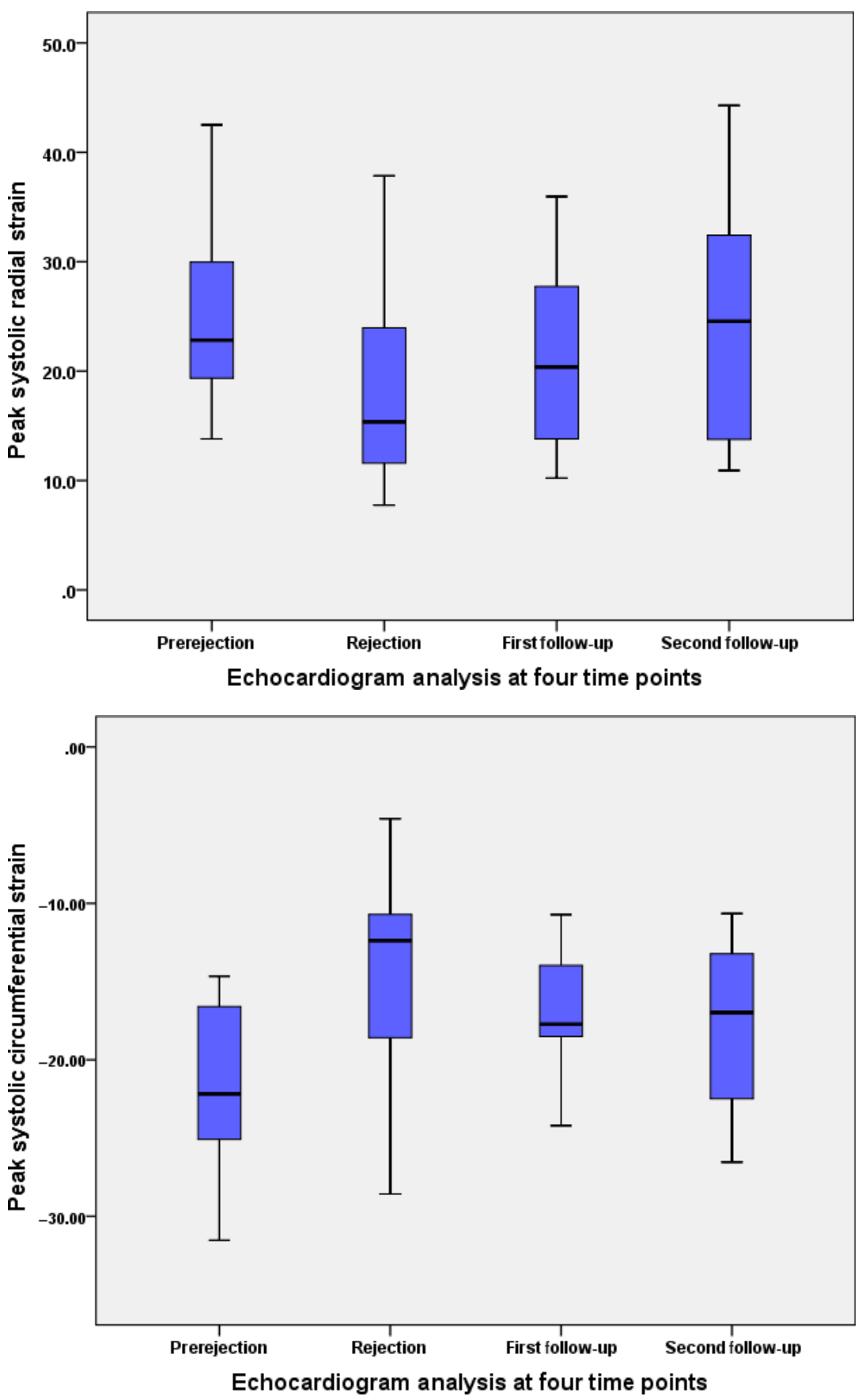

Fig. 2. Peak systolic radial strain at four time points: baseline, rejection, first and second follow-up. First followup: 1.5 months (0.5-5 months) from rejection episode [Median (range)]. Second follow-up: six months (4-12 months) from rejection episode [Median (range)].
Fig. 3. Peak systolic circumferential strain at four time points: baseline, rejection, first and second follow-up. First follow-up: 1.5 months $(0.5-$ 5 months) from rejection episode [Median (range)]. Second follow-up: six months (4-12 months) from rejection episode [Median (range)]. 
A recent study compared strain and strain rate patterns using vector velocity imaging in 28 pediatric patients following heart transplant, who had no evidence of rejection, and 28 age- and gender-matched normal controls. The longitudinal strain was decreased $(22.14 \%$ vs. 17.21$)$ in heart transplant recipients, one yr following transplant even without rejection. There was no significant difference in the peak global circumferential strain pattern between the two groups (20.28\% vs. $20.79 \%)$ (19). Consistent with these results, in our study, even at baseline, the peak systolic longitudinal and peak systolic radial strain were lower, compared to published normal values (13). This was despite our careful selection of subjects a year or more following transplant, beyond the period of intensive immunosuppressant therapy and selecting the baseline during a six-month rejection-free duration. This may be due to the allograft ischemic time, use of cardioplegia, denervation of heart and reperfusion injury, prior rejection episodes, or chronic immunosuppressant medications in heart transplant recipients (20). Nonetheless, strain profiles in individual patients deteriorated during a rejection episode, compared to baseline, and may be useful biomarkers of rejection. In our cohort, systolic strain indices appeared to improve following treatment of rejection during a mean follow-up duration of about six months, although this did not reach statistical significance. Acute rejection is associated with accumulation of inflammatory cells, edema of interstitial tissues, and damage to myocardial fibers. These changes may take many months to resolve. We speculate that strain imaging abnormalities appear early in the course of a rejection episode prior to changes in conventional systolic and diastolic functional indices and persist for long, even at a time when hemodynamic parameters on catheterization have normalized.

Conventional ECHO parameters that have been investigated for their value in rejection surveillance include fractional shortening, LV wall thickness and mass, LV diastolic dimensions, wall stress and stress velocity, and diastolic parameters such as mitral valve Doppler inflow, tissue Doppler velocities, and MPI with inconsistent results (21-28). In a pediatric study by Moran et al., higher mitral E-wave amplitude was significantly associated with acute rejection, while there was no significant correlation between systolic ECHO parameters and rejection (21). In contrast, a study by Boyd et al. showed that the mitral $\mathrm{E}$, $\mathrm{A}$ velocities and $\mathrm{E} / \mathrm{A}$ ratio did not vary with biopsy scores (23). Another study by Pellicelli et al. showed a significant decrease in mitral $\mathrm{E}$ velocity with severe rejection alone,

Table 3. Comparison of the ECHO and hemodynamic parameters between baseline, rejection and two follow up points

\begin{tabular}{|c|c|c|c|c|c|}
\hline Parameter & $\begin{array}{l}\text { Baseline } \\
\text { Mean (SD) }\end{array}$ & $\begin{array}{l}\text { Rejection } \\
\text { Mean (SD) }\end{array}$ & $\begin{array}{l}\text { F/u } 1 \\
\text { Mean (SD) }\end{array}$ & $\begin{array}{l}\text { F/u } 2 \\
\text { Mean (SD) }\end{array}$ & $\mathrm{p}$ \\
\hline \multicolumn{6}{|l|}{ ECHO parameters } \\
\hline Fractional shortening (\%) & $34(3.8)$ & $31.5(9.3)$ & $33.1(5.8)$ & $32.4(4.3)$ & 0.3 \\
\hline LV mass $\left(\mathrm{g} / \mathrm{ht}^{2.7}\right)$ & 34 (11.2) & $47.5(18.7)$ & $38.7(12.5)$ & 40 (13) & 0.03 \\
\hline LV posterior wall thickness $(\mathrm{cm})$ & $0.7(0.1)$ & $0.8(0.3)$ & $0.7(0.2)$ & $0.7(0.2)$ & 0.1 \\
\hline Mitral E & $97(20)$ & $103(34)$ & $99(34)$ & $99(25)$ & 0.6 \\
\hline Mitral A & 48 (13) & $50(7)$ & $56(13)$ & $53(13)$ & 0.7 \\
\hline Septal E' & $10.6(2.4)$ & $10.5(2.1)$ & $9(6)$ & $10(2)$ & 0.9 \\
\hline Septal A' & $5.2(1.9)$ & $4.9(2)$ & $4.5(2.4)$ & $4.2(1.5)$ & 0.8 \\
\hline Lateral E' & $14.7(3.9)$ & $17(10)$ & $15.8(3.6)$ & $13(3)$ & 0.5 \\
\hline Lateral A' & $6.5(2.1)$ & $6.1(1.9)$ & $6.5(5.6)$ & $5.3(2.3)$ & 0.7 \\
\hline $\mathrm{E} / \mathrm{A}$ & $2.1(0.5)$ & $2(0.5)$ & $1.8(0.5)$ & $2(0.6)$ & 0.7 \\
\hline Septal E/E' & $8.8(1.9)$ & $10.4(3.6)$ & $28.7(37.2)$ & $10.2(3)$ & 0.3 \\
\hline Septal S' & $6(1.5)$ & $5.4(1.4)$ & $5.7(0.5)$ & $6(2.1)$ & 0.4 \\
\hline Lateral E/E' & $6.3(1.2)$ & $7.3(3.6)$ & $6.7(2.3)$ & $7.9(3.4)$ & 0.4 \\
\hline Lateral S' & $7.8(1.9)$ & $7(1.5)$ & $6.6(1)$ & $6.8(1.9)$ & 0.3 \\
\hline LV MPI & $1.5(0.3)$ & $1.6(0.5)$ & $1.5(0.3)$ & $1.6(0.3)$ & 0.5 \\
\hline \multicolumn{6}{|l|}{ Hemodynamic parameters } \\
\hline \multirow[t]{2}{*}{ mRAP, mmHg } & $4.5(2.4)$ & $8.5(5.1)$ & $5.3(3.3)$ & $4.9(2.6)$ & $0.001^{*}$ \\
\hline & & & & & $0.03^{* *}$ \\
\hline \multirow[t]{2}{*}{ mPCWP, mmHg } & $8.1(1.9)$ & $11(5)$ & $8.3(3.3)$ & $7.5(3)$ & $0.04^{*}$ \\
\hline & & & & & $0.04^{* *}$ \\
\hline
\end{tabular}

F/u 1: First follow-up: 1.5 months (0.5-5 months) from rejection episode [Median (range)].

F/u 2: Second follow-up: six months (4-12 months) from rejection episode [Median (range)].

Numbers in bold are statistically significant results.

*Significant difference between baseline and rejection; **significant difference between rejection and second follow-up. 
compared to patients without rejection (24). Behera et al. studied multiple tissue Doppler derived diastolic indices in children with biopsypositive and clinical rejection and found that mitral and tricuspid valve $\mathrm{E} / \mathrm{E}^{\prime}<5.0$ had $93 \%$ and $89 \%$ negative predictive values, respectively, for rejection; the sensitivity, specificity, and positive predictive values were low (27). Because of significant overlap found in TDI velocities between patients with and without rejection, Lunze et al. defined non-rejection as preservation of left ventricular S' and A' velocities (29). In our study, we did not find significant differences in diastolic indices from baseline to rejection. Nor was there a change in the LV MPI from baseline to rejection, although there is some evidence to show that increased MPI correlates with biopsy-proven rejection in the pediatric population $(25,26)$.

We did see a significant increase in LV mass during an episode of rejection, consistent with some other reports (30-32). Sagar et al. studied 12 episodes of rejection and found that the LV mass increased significantly by $139 \%$, compared to the post-transplant prerejection LV mass. In contrast, LV mass did not increase without rejection $(p>0.2)$ (30). Santos-Ocampo compared the LV mass index in 12 infants younger than 20 months of age with grade $3 \mathrm{~A}$, or higher rejection was significantly higher, compared to LV mass index two wk after resolution of the rejection and compared to controls not in rejection. Kawauchi et al. further showed that increase in LV mass was proportional to the number of rejection episodes (32).

\section{Limitations}

Because of the retrospective design of the study, we computed strain and strain rate from the limited ECHO images that were obtained as part of the clinical routine protocol. Hence, our measurements were limited to six LV segments, although abnormalities in these segments are probably representative of the global strain abnormality and such a method has been previously used. Additionally, the lower frame rate of the stored DICOM images can affect the accuracy of strain and especially strain rate measurements. The small sample size precluded drawing meaningful ROC curves and determining cutoff points for the diagnosis of acute rejection. Owing to small numbers, we did not analyze symptomatic and AMR patients separately. Based on our data, it is not possible to know whether very mild grade asymptomatic rejection would show detectable strain abnormalities. Our results need to be validated in a large population. Nonetheless, to our knowledge, this study was a novel attempt to investigate the pattern of strain and strain rate during rejection using speckle tracking in this population.

\section{Conclusion}

This observational study demonstrates that peak systolic radial, longitudinal, and circumferential strain by speckle tracking are abnormal during an acute rejection episode in pediatric heart transplant recipients. These changes are seen in the absence of significant changes in other established ECHO measures of systolic and diastolic function and LV MPI. Serial surveillance by strain imaging using speckle tracking echocardiography may aid clinicians in the diagnosis of acute rejection.

\section{Conflict of interest}

No conflict of interest or grant for this project.

\section{References}

1. Boucek MM, Waltz DA, Edwards LB, et al. Registry of the International Society for Heart and Lung Transplantation: Ninth official pediatric heart transplantation report-2006. J Heart Lung Transplant 2006: 25: 893-903.

2. Sun JP, Abdalla IA, Asher CR, et al. Non invasive evaluation of orthotopic heart transplant rejection by echocardiography. J Heart Lung Transplant 2005: 24: 160-165.

3. Hosenpud JD, Bennett LE, Keck BM, Boucek MM, Novick RJ. The Registry of the International Society for Heart and Lung Transplantation: Seventeenth official report-2000. J Heart Lung Transplant 2000: 19: 909-931.

4. Baraldi-Junkins C, Levin HR, Kasper eK, Rayburn BK, Herlowitz A, Baughman KL. Complications of endomyocardial biopsy in heart transplant patients. J Heart Lung Transplant 1993: 12: 63-67.

5. Rosenthal DN, Chin C, Nishimura K, et al. Identifying cardiac transplant rejection in children: Diagnostic utility of echocardiography, right heart catheterization and endomyocardial biopsy data. J Heart Lung Transplant 2004: 23: 323-329.

6. Blessberger H, Binder T. Two dimensional speckle tracking echocardiography: Basic principles. Heart 2010: 96: 716-722.

7. Urheim S, Edvardsen T, Torp H, Angelsen B, Smiseth OA. Myocardial strain by Doppler echocardiography. Validation of a new method to quantify regional myocardial function. Circulation 2000: 102: 1158-1164.

8. Shimoni S, Gendelman G, Ayzenberg O. Differential effects of coronary artery stenosis on myocardial function: The value of myocardial strain analysis for the detection of coronary artery disease. J Am Soc Echocardiogr 2011: 24: 748-757.

9. Mori K, Hayabuchi Y, Inoue M, et al. Myocardial strain imaging for early detection of cardiac involvement in patients with Duchenne's progressive muscular dystrophy. Echocardiography 2007: 24: 598-608.

10. Poterucha JT, Kutty S, Lindquist RK, Li L, Eidem BW. Changes in left ventricular longitudinal strain with anthracycline chemotherapy in adolescents precede subsequent 
decreased left ventricular ejection fraction. J Am Soc Echocardiogr 2012: 25: 733-740.

11. Barbosa Ja, Mota CC, Simões E, Silva AC, Nunes Mdo C, Barbosa MM. Assessing pre-clinical ventricular dysfunction in obese children and adolescents: The value of speckle tracking imaging. Eur Heart J Cardiovasc Imaging 2013: 14: 882-889.

12. Stewart S, Winters GL, Fishbein MC, et al. Revision of the 1990 working formulation for the standardization of nomenclature in the diagnosis of heart rejection. J Heart Lung Transplant 2005: 24: 1710-1720.

13. Lorch SM, Ludomirsky A, Singh GK. Maturational and growth-related changes in left ventricular longitudinal strain and strain rate measured by two-dimensional speckle tracking echocardiography in healthy pediatric population. J Am Soc Echocardiogr 2008: 21: 1207-1215.

14. Pieper GM, Shah A, Harmann L, Cooley BC, Ionova iA, Migrino RQ. Speckle tracking 2 dimensional strain echocardiography: A new noninvasive imaging tool to evaluate acute rejection in cardiac transplantation. J Heart Lung Transplant 2010: 29: 1039-1046.

15. Sera F, Kato TS, Farr M, et al. Left ventricular longitudinal strain by speckle-tracking echocardiography is associated with treatment-requiring cardiac allograft rejection. J Card Fail 2014: 20: 359-364.

16. Kato TS, Oda N, Hashimura $\mathrm{K}$, et al. Strain rate imaging would predict sub clinical acute rejection in heart transplant recipients. Eur J Cardiothorac Surg 2010: 37: 1104 1110.

17. Roshanali F, Mandegar MH, Bagheri $\mathbf{J}$, et al. Echo rejection score: New echocardiographic approach to diagnosis of heart transplant rejection. Eur J Cardiothorac Surg 2010: 38: 176180.

18. Storaa C, Aberg P, Lind B, Brodin LA. Effect of angular error on tissue Doppler velocities and strain. Echocardiography 2003: 20: 581-587.

19. Kailin JA, Miyamoto SD, Younoszai AK, Landeck BF. Longitudinal myocardial deformation is selectively decreased after pediatric cardiac transplantation: A comparison of children 1 year after transplantation with normal subjects using velocity vector imaging. Pediatr Cardiol 2012: 33: 749-756.

20. Eroglu E, Herbots L, Van Cleemput J, et al. Ultrasonic strain/strain rate imaging-a new clinical tool to evaluate the transplanted heart. Eur J Echocardiogr 2005: 6: 186-195.

21. Moran AM, Lipshultz SE, Rifai N, et al. Non-invasive assessment of rejection in pediatric transplant patients:
Serologic and echocardiographic prediction of biopsy-proven myocardial rejection. J Heart Lung Transplant 2000: 19: 756764.

22. Neuberger S, Vincent RN, Doelling N, et al. Comparison of quantitative echocardiography with endomyocardial biopsy to define myocardial rejection in pediatric patients after cardiac transplantation. Am J Cardiol 1997: 79: 447-450.

23. Boyd Sy, Mego DM, Khan NA, Rubal BJ, Gilbert TM. Doppler echocardiography in cardiac transplant patients: Allograft rejection and its relationship to diastolic function. J Am Soc Echocardiogr 1997: 10: 526-531.

24. Pellicelli AM, Cosial JB, Ferranti E, Gomez A, Borgia MC. Alteration of left ventricular filling evaluated by Doppler echocardiography as a potential marker of acute rejection in orthotopic heart transplant. Angiology 1996: 47: 35-41.

25. Leonard GT Jr, Fricker FJ, Pruett D, Harker K, Williams $\mathrm{B}$, Schowengerdt KO Jr. Increased myocardial performance index correlates with biopsy-proven rejection in pediatric heart transplant recipients. J Heart Lung Transplant 2006: 25: 61-66.

26. Mooradian SJ, Goldberg CS, Crowley DC, Ludomirsky A. Evaluation of a noninvasive index of global ventricular function to predict rejection after pediatric cardiac transplantation. Am J Cardiol 2000: 86: 358-360.

27. Behera SK, Trang J, Feeley BT, Levi DS, Alejos JC, Drant $\mathrm{S}$. The use of Doppler tissue imaging to predict cellular and antibody-mediated rejection in pediatric heart transplant recipients. Pediatr Transplant 2008: 12: 207-214.

28. Sachdeva R, Malik S, Seib PM, Frazier EA, Cleves MA. Doppler tissue imaging and catheter-derived measures are not independent predictors of rejection in pediatric heart transplant recipients. Int J Cardiovasc Imaging 2011: 27: 947-954.

29. Lunze FI, Colan SD, Gauvreau K, et al. Tissue Doppler imaging for rejection surveillance in pediatric heart transplant recipients. J Heart Lung Transplant 2013: 32: 1027-1033.

30. Sagar KB, Hastillo A, Wolfgang TC, Lower RR, Hess ML. Left ventricular mass by $\mathrm{M}$ mode echocardiography in cardiac transplant patients with acute rejection. Circulation 1981: 64: II217-II220.

31. Santos-Ocampo SD, Sekarski TJ, Saffitz JE, et al. Echocardiographic characteristics of biopsy proven cellular rejection in infant heart transplant recipients. J Heart Lung Transplant 1996: 15: 25-34.

32. Kawauchi M, Bouceck MM, Gundry SR. Changes in left ventricular mass with rejection after heart transplantation in infants. J Heart Lung Transplant 1992: 11: 99-102. 